\title{
Perceptions and behaviours towards high body weight among adults in Northeast China
}

\author{
Chunshi Gao, Xin Lv, Yutian Yin, Yuanyuan Song, Peng Zhang, Rui Wang, Lingling Jiang, \\ Yuhan Wang, Yaqin Yu and Bo Li* \\ Department of Epidemiology and Biostatistics, Jilin University School of Public Health, 1163 Xinmin Street, \\ Changchun, Jilin 130021, People's Republic of China
}

Submitted 21 July 2016: Final revision received 2 March 2017: Accepted 3 March 2017: First published online 5 April 2017

\begin{abstract}
Objective: To determine the prevalence of high weight at different characteristics, understand the perceptions and behaviours towards high body weight, and determine potential influencing factors of body weight misperception among high-weight adults in Jilin Province.

Design: A cross-sectional survey with complex sampling design was conducted. We described the prevalence and perception of high body weight.

Setting: Northeast China in 2012.

Subjects: Adults ( $n 20$ 552) aged 18-79 years.

Results: Of overweight individuals, $37.4 \%$ considered themselves as 'normal weight', $4.8 \%$ reported themselves as being 'very thin' and only $53 \cdot 1 \%$ were aware of their own weight being 'overweight'. About $1.8 \%$ of both male and female obese individuals perceived themselves as 'very thin'. Only $29.1 \%$ of obese people thought of themselves as 'too fat'. Nearly $30.0 \%$ of centrally obese men and women perceived that their waist circumference was about right and they were of 'normal weight'; $5.7 \%$ of the centrally obese even perceived themselves as being 'very thin'. Only 51.8 and $12.5 \%$ of centrally obese individuals reported themselves to be 'overweight' or 'too fat'. Body weight misperception was more common in rural residents (OR; 95\% CI: 1.340; 1.191, 1.509). The prevalence of body weight misperception increased with age (middle age: 1.826; 1.605, 2.078; old people: $3 \cdot 101 ; 2 \cdot 648,3.632$ ) and declined with increased education level (junior middle school: $0.628 ; 0.545,0.723$; senior middle school: $0.498 ; 0.426$, 0.583; undergraduate and above: 0.395; 0.320, 0.487).

Conclusions: Body weight misperception was common among adults from Jilin Province.
\end{abstract}

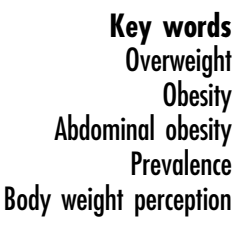

The rising prevalence rates of overweight and obesity have been regarded as a global pandemic ${ }^{(1-5)}$. A study in the USA showed that the high obesity prevalence could lead to a fall in life expectancy in the future ${ }^{(6)}$. Considering the increased risk of obesity-related diseases, a voluntary target with the purpose of stopping the rise of obesity by 2025 was introduced in WHO member states ${ }^{(7)}$. The WHO provides advocacy to help lower the prevalence of overweight in all populations ${ }^{(8,9)}$. According to the nutrition and chronic disease status reports of Chinese residents in 2015 , the prevalences of overweight and obesity among adults were $7 \cdot 1$ and $4.5 \%$ in 2002, respectively. However, in a short span of 10 years, the prevalence rates had almost doubled in 2012 (overweight: 11.9\%; obesity: 9.6\%). The overweight and obesity prevalences in Chinese children increased rapidly from $22 \cdot 8 \%$ in 2002 to $30 \cdot 1 \%$ in 2012 and from $2 \cdot 1 \%$ in 2002 to $6 \cdot 4 \%$ in 2012 , respectively ${ }^{(10)}$. It was reported that the overall obesity prevalence was $3.3 \%$ in China in 1992. A rapid growth in the prevalences of overweight and obesity was perceived. The prevalence rates of overweight and obesity will continue to increase if no attention is paid to them and no effective intervention measures is taken ${ }^{(11)}$.

The increased overweight and obesity prevalences and the marked shift in lifestyle-related non-communicable disease patterns in China over the past few decades may be due to rapid economic growth and urbanization as well as overnutrition characterized by increased intakes of high-fat/high-energy foods ${ }^{(4,12-15)}$.

Evidence indicates that the prevalences of overweight and obesity in children and adolescents worldwide have reached worrying levels ${ }^{(16,17)}$. Obese children are more 
likely to have psychological health issues due to low self-esteem compared with non-obese peers ${ }^{(18)}$. Similarly, the lifestyle-related transformation has largely contributed to the increased prevalence rates of overweight and obesity among children and youths ${ }^{(19)}$. A meta-analysis conducted on the prevalence of overweight/obesity in Portuguese children showed that during the last decade, $30.3 \%$ of Portuguese children were overweight or obese ${ }^{(20)}$.

Besides the high prevalences of overweight, obesity and abdominal obesity as well as the threat of obesity-related diseases, body weight misperception is also common among adults. Many studies conducted in different countries and populations have demonstrated that a considerable number of adults do not perceive their body weight correctly ${ }^{(21-26)}$. A study in Sri Lanka showed that $44.7 \%$ of overweight females and almost two-thirds of overweight males reported themselves to be 'right weight', and $7 \cdot 6 \%$ of overweight females and $4.1 \%$ of overweight males considered themselves to be 'underweight'. Over one-third of obese individuals (both males and females) even thought themselves to be 'underweight' or 'right weight'. Nearly $32 \%$ of abdominally obese individuals perceived that their waist circumference was within the normal range. Only $63.6 \%$ thought of themselves as 'overweight' or 'obese' and tried to lose weight or take advice from professionals ${ }^{(27)}$.

Our study aimed to determine the prevalence of high weight at different characteristics, understand the perceptions and behaviours towards high body weight, and determine potential influencing factors of body weight misperception among adults in Jilin Province.

\section{Methods}

\section{Participants}

The present study was part of a large cross-sectional survey of chronic diseases in Jilin Province in 2012. Using the method of multistage stratified cluster sampling (the stratifying process has been reported previously ${ }^{(28)}$ ), the study was conducted among residents aged 18-79 years in nine regions per administrative division (Changchun, Jilin, Siping, Liaoyuan, Tonghua, Baishan, Songyuan, Baicheng and Yanbian). A total of 23050 individuals were recruited and 21435 of them completed the survey (response rate 84.9\%). A total of 20552 individuals (5645 in Changchun, 3160 in Jilin, 2779 in Siping, 1302 in Liaoyuan, 1263 in Tonghua, 957 in Baishan, 2217 in Songyuan, 1483 in Baicheng and 1746 in Yanbian) who had detailed information from the physical examination were chosen for the present study. The percentages of males and females were $47 \cdot 0 \%$ ( $n$ 9651) and 53.0\% ( $n 10$ 901), respectively. To make the sample more representative, the data analyses were weighted by post-stratification adjustment according to the factors of age, area and gender, using the 2010 China (Jilin Province) Population Census.

\section{Data collection}

We used the method of questionnaire investigation (sociodemographic characteristics and health-related information) and body measurements (such as height, weight and waist circumference). Sociodemographic characteristics included gender, residence (rural/urban), age, area, ethnicity, education level, marital status, occupation and income class, and health-related information included perceptions and behaviours towards high weight. All investigators had received uniform training. We calibrated the measurement instruments with a common standard before use ${ }^{(29)}$.

\section{Measurements}

The physical examination included the measurement of height, weight, waist and hip circumferences. The participants were asked to take off their shoes and wear light clothes, and the indices were recorded to the nearest $0 \cdot 1 \mathrm{~cm}$ or $0 \cdot 1 \mathrm{~kg}$.

\section{Definitions}

We calculated BMI as weight $/ \mathrm{height}^{2}\left(\mathrm{~kg} / \mathrm{m}^{2}\right)$. All participants were categorized as normal weight $(\mathrm{BMI}=18.5$ to $25 \mathrm{~kg} / \mathrm{m}^{2}$ ), underweight $\left(\mathrm{BMI}<18.5 \mathrm{~kg} / \mathrm{m}^{2}\right)$, overweight $\left(\mathrm{BMI}=25 \cdot 0\right.$ to $\left.30 \cdot 0 \mathrm{~kg} / \mathrm{m}^{2}\right)$ or obese $\left(\mathrm{BMI}>30 \cdot 0 \mathrm{~kg} / \mathrm{m}^{2}\right)^{(30)}$. Waist circumference $>94 \mathrm{~cm}$ in males and $>80 \mathrm{~cm}$ in females was defined as abdominal obesity ${ }^{(31)}$.

\section{Data analysis}

In addition to the complex sampling design, poststratification adjustment according to the distribution of gender and age groups was also used for our study ${ }^{(32)}$. EpiData 3.1 software was used for establishment of the database and the statistical analyses were carried out using the IBM SPSS Complex Samples add-on module with IBM SPSS Statistics Version 21.0. We used $\chi^{2}$ tests to compare the distribution of high weight at different characteristics and multiple logistic regression analyses were used to explore the association between sociodemographic factors and body weight misperception. $P<0.05$ was considered to be statistically significant.

\section{Results}

The prevalence of high weight at different characteristics by gender is shown in Table 1 .

Among males, the prevalence of overweight, obesity and abdominal obesity in urban residents was higher than that in rural residents; however, it is interesting to note that the results in females were precisely the opposite. Among females, those of Han ethnicity were more likely to develop high body weight compared with other ethnicities. Males with a higher level of education (undergraduate and above) had a higher prevalence of high weight than those with a lower level of education, 
Table 1 The prevalence of high weight at different characteristics* by gender among adults ( $n 20552)$ aged $18-79$ years from Jilin Province, Northeast China, 2012

\begin{tabular}{|c|c|c|c|c|c|c|c|c|c|c|c|c|c|c|c|c|c|c|}
\hline \multirow[b]{3}{*}{ Characteristic } & \multicolumn{6}{|c|}{ Overweight participants } & \multicolumn{6}{|c|}{ Obese participants } & \multicolumn{6}{|c|}{ Abdominally obese participants } \\
\hline & \multicolumn{2}{|c|}{ Male } & \multicolumn{2}{|c|}{ Female } & \multicolumn{2}{|c|}{ Total } & \multicolumn{2}{|c|}{ Male } & \multicolumn{2}{|c|}{ Female } & \multicolumn{2}{|c|}{ Total } & \multicolumn{2}{|c|}{ Male } & \multicolumn{2}{|c|}{ Female } & \multicolumn{2}{|c|}{ Total } \\
\hline & $n$ & $\%$ & $n$ & $\%$ & $n$ & $\%$ & $n$ & $\%$ & $n$ & $\%$ & $n$ & $\%$ & $n$ & $\%$ & $n$ & $\%$ & $n$ & $\%$ \\
\hline Residence & & & & & & & & & & & & & & & & & & \\
\hline Rural & 1567 & $31 \cdot 1$ & 1927 & $32 \cdot 0$ & 3494 & 31.5 & 672 & $15 \cdot 3$ & 851 & 13.8 & 1523 & 14.6 & 2034 & 41.6 & 2788 & $44 \cdot 1$ & 4822 & $42 \cdot 8$ \\
\hline Urban & 1901 & $36 \cdot 8$ & 1802 & 28.8 & 3703 & 33.0 & 818 & $17 \cdot 0$ & 716 & 11.9 & 1534 & 14.6 & 2581 & $50 \cdot 4$ & 2481 & 39.2 & 5062 & $45 \cdot 0$ \\
\hline$x^{2}$ & 36 & & 14 & & & & & & & & & & & & & & & \\
\hline$\hat{P}$ & & & & & & & & & & & & & & & & & & \\
\hline Age & & & & & & & & & & & & & & & & & & \\
\hline Youth & 1363 & 30.9 & 1126 & $22 \cdot 7$ & 2489 & $27 \cdot 0$ & 729 & $17 \cdot 7$ & 435 & $9 \cdot 1$ & 1164 & 13.6 & 1811 & $42 \cdot 3$ & 1267 & $26 \cdot 1$ & 3078 & 34.7 \\
\hline Middle age & 1454 & 41.2 & 1736 & 39.6 & 3190 & $40 \cdot 4$ & 535 & $15 \cdot 2$ & 753 & $17 \cdot 1$ & 1288 & $16 \cdot 1$ & 1889 & $53 \cdot 6$ & 2506 & $56 \cdot 4$ & 4395 & $55 \cdot 0$ \\
\hline Old people & 651 & 34.9 & 867 & 40.2 & 1518 & 37.6 & 226 & $12 \cdot 2$ & 379 & 18.0 & 605 & $15 \cdot 1$ & 915 & $50 \cdot 1$ & 1496 & 69.0 & 2411 & 59.7 \\
\hline$x^{2}$ & & & & & & & & & & & & & & & & & & \\
\hline$\hat{P}$ & & & & & & & & & & & & & & & & & & \\
\hline Area & & & & & & & & & & & & & & & & & & \\
\hline Central & 2124 & 34.2 & 2275 & $29 \cdot 0$ & 4399 & 31.7 & 870 & 15.5 & 968 & $12 \cdot 3$ & 1838 & 14.0 & 2713 & 44.6 & 3177 & $39 \cdot 2$ & 5890 & $42 \cdot 0$ \\
\hline Eastern & 732 & $35 \cdot 3$ & 709 & 32.6 & 1441 & 34.0 & 315 & 16.5 & 285 & 13.8 & 600 & $15 \cdot 1$ & 949 & $47 \cdot 3$ & 960 & 44.4 & 1909 & 45.9 \\
\hline Western & 612 & 33.3 & 745 & 31.6 & 1357 & 32.5 & 305 & 18.6 & 314 & $13 \cdot 1$ & 619 & $15 \cdot 9$ & 953 & 52.6 & 1132 & $45 \cdot 5$ & 2085 & 49.1 \\
\hline$x^{2}$ & & & & & & & & & & & & & & & & & & \\
\hline$\hat{P}$ & & & & & & & & & & & & & & & & & & \\
\hline Ethnicity & & & & & & & & & & & & & & & & & & \\
\hline Han & 3202 & 34.2 & 3448 & 30.3 & 6650 & 32.3 & 1384 & $16 \cdot 2$ & 1646 & 13.1 & 2848 & 14.7 & 4279 & 46.5 & 4912 & $42 \cdot 0$ & 9191 & $44 \cdot 3$ \\
\hline Others & 266 & 35.8 & 281 & 29.4 & 547 & 32.5 & 106 & 16.3 & 103 & 9.4 & 209 & 12.8 & 336 & 47.0 & 357 & 34.8 & 693 & 40.8 \\
\hline$x^{2}$ & & & & & & & & & & & & & & & & & & \\
\hline$\hat{P}$ & & & & & & & & & & & & & & & & & & \\
\hline Education & & & & & & & & & & & & & & & & & & \\
\hline Primary school and below & 710 & 31.4 & 1482 & 36.8 & 2192 & 34.6 & 247 & $12 \cdot 1$ & 701 & 17.5 & 948 & $15 \cdot 3$ & 839 & 37.7 & 2362 & 57.8 & 3201 & $49 \cdot 6$ \\
\hline Junior middle school & 1026 & 31.9 & 1012 & 30.3 & 2038 & $31 \cdot 1$ & 457 & $16 \cdot 5$ & 446 & 13.7 & 903 & $15 \cdot 2$ & 1410 & 45.5 & 1398 & $40 \cdot 2$ & 2808 & 43.0 \\
\hline Senior middle school & 1052 & 36.5 & 837 & 29.3 & 1889 & 33.4 & 446 & $16 \cdot 3$ & 313 & 11.5 & 759 & $14 \cdot 2$ & 1424 & $49 \cdot 3$ & 1062 & 37.0 & 2486 & 44.0 \\
\hline Undergraduate and above & 680 & 37.2 & 398 & 21.4 & 1078 & 30.1 & 340 & 19.5 & 107 & 5.5 & 447 & 13.2 & 942 & 52.0 & 447 & 23.7 & 1389 & 39.1 \\
\hline$x^{2}$ & & & & & & & & & & & $1 c$ & & 10 & & 70 & & 11 & \\
\hline$\stackrel{A}{P}$ & & & & & & & & & & & & & & & & & & \\
\hline Marital status & & & & & & & & & & & & & & & & & & \\
\hline Married & 3090 & $36 \cdot 7$ & 3307 & 32.8 & 6397 & 34.8 & 1295 & $16 \cdot 9$ & 1389 & 13.8 & 2684 & $15 \cdot 4$ & 4126 & $50 \cdot 2$ & 4620 & $44 \cdot 3$ & 8746 & $47 \cdot 3$ \\
\hline Unmarried & 235 & $22 \cdot 3$ & 61 & 8.2 & 296 & $16 \cdot 7$ & 153 & 14.7 & 25 & 3.3 & 178 & $10 \cdot 2$ & 314 & 30.2 & 73 & 11.0 & 387 & $22 \cdot 6$ \\
\hline Separated/divorced & 74 & 43.4 & 59 & 31.1 & 133 & 38.0 & 24 & 12.4 & 27 & 13.0 & 51 & 12.7 & 92 & $48 \cdot 8$ & 80 & 41.1 & 172 & 45.4 \\
\hline Widowed & 69 & 30.5 & 302 & 39.3 & 371 & 37.0 & 18 & 7.9 & 126 & 17.2 & 144 & 14.8 & 83 & 36.9 & 496 & 63.9 & 579 & 56.9 \\
\hline$x^{2}$ & & & & & & & & & & & & & & & & & & \\
\hline${ }_{P}^{X}$ & & & & & & & & & & & & & & & & & & \\
\hline Occupation & & & & & & & & & & & & & & & & & & \\
\hline Unemployed & 228 & $32 \cdot 1$ & 687 & 32.0 & 915 & 32.0 & 125 & $19 \cdot 7$ & 341 & $16 \cdot 3$ & 466 & 17.4 & 349 & $49 \cdot 7$ & 1090 & 49.4 & 1439 & 49.5 \\
\hline Mental & 812 & 36.4 & 579 & 23.3 & 1391 & 30.0 & 379 & $18 \cdot 2$ & 173 & 6.5 & 552 & 12.5 & 1146 & $52 \cdot 2$ & 656 & $26 \cdot 4$ & 1802 & 39.5 \\
\hline Manual & 2076 & 33.1 & 1855 & 30.4 & 3931 & 32.0 & 837 & $15 \cdot 1$ & 790 & 13.1 & 1627 & $14 \cdot 3$ & 2581 & $42 \cdot 3$ & 2572 & 40.5 & 5153 & 41.6 \\
\hline Retired & 352 & 39.1 & 608 & $42 \cdot 1$ & 960 & 40.9 & 149 & $15 \cdot 9$ & 263 & 19.5 & 412 & 18.0 & 539 & 59.5 & 951 & $66 \cdot 3$ & 1490 & 63.4 \\
\hline$x^{2}$ & & & & & & & & & & & & & & & & & & \\
\hline${ }_{P}^{X}$ & & & & & & & & & & & & & & & & & & \\
\hline Income class & & & & & & & & & & & & & & & & & & \\
\hline Low & 557 & $32 \cdot 1$ & 943 & 35.5 & 1500 & 33.9 & 221 & $15 \cdot 1$ & 424 & $15 \cdot 9$ & 645 & $15 \cdot 5$ & 722 & $43 \cdot 1$ & 1457 & 53.5 & 2179 & 48.8 \\
\hline Middle & 1130 & 33.7 & 1214 & 29.7 & 2344 & 31.7 & 476 & $15 \cdot 5$ & 480 & $12 \cdot 2$ & 956 & 13.8 & 1500 & $45 \cdot 3$ & 1650 & 39.6 & 3150 & 42.4 \\
\hline Higher middle & 790 & 36.5 & 541 & $26 \cdot 4$ & 1331 & $32 \cdot 2$ & 341 & $17 \cdot 4$ & 191 & 9.2 & 532 & 13.9 & 1046 & $49 \cdot 1$ & 692 & $32 \cdot 3$ & 1738 & $42 \cdot 0$ \\
\hline High & 440 & $36 \cdot 1$ & 235 & 22.8 & 675 & 30.9 & 237 & 20.5 & 91 & 9.0 & 328 & $16 \cdot 1$ & 626 & $52 \cdot 2$ & 317 & 30.4 & 943 & 43.8 \\
\hline Lower middle & 551 & 32.5 & 796 & 33.9 & 1347 & 33.2 & 215 & 13.5 & 381 & $16 \cdot 2$ & 596 & $15 \cdot 0$ & 721 & 43.4 & 1153 & 47.7 & 1874 & $45 \cdot 7$ \\
\hline$x^{2}$ & & & & & & & & & & & & & & & & & & \\
\hline$\hat{P}$ & & & & & & & & & & & & & & & & & & \\
\hline
\end{tabular}

${ }^{*}$ The numbers are unweighted, but the percentages are weighted. Complex weighted computation was used in the statistical analysis. 
but the prevalence overall in females showed a downward tendency along with increasing education levels. Retired people had a higher chance of having high body weight than those who were not retired. In addition, the differences according to age, area, marital status and income class were of statistical significance.

Body weight perception, management and changes in the past 12 months, as well as the related lifestyles and behaviours of weight-control practices among adults in Jilin Province, are shown in Table 2.

Body weight misperception was common among adults in Jilin Province. Among those who were overweight, $44.5 \%$ of males and $28.9 \%$ of females considered themselves of 'normal weight'. Moreover, $4.6 \%$ of overweight men and $5.0 \%$ of overweight women reported themselves being 'very thin'. Only $48.1 \%$ of overweight men and $59.2 \%$ of overweight women were aware of their weight being 'overweight, and $4.7 \%$ of overweight participants considered themselves 'too fat'.

About $1.8 \%$ of both male and female obese participants perceived themselves as 'very thin'. Only $24.7 \%$ of obese men and $35.0 \%$ of obese women were aware of themselves being 'too fat', and $10 \cdot 0 \%$ obese participants thought that they had 'normal weight'.

Nearly $30.0 \%$ of centrally obese men and women perceived that their waist circumference was about right and they were of normal weight, and $5.7 \%$ of centrally obese men and women even perceived themselves to be 'very thin'. Only 51.8 and $12.5 \%$ of centrally obese participants reported themselves as 'overweight' and 'too fat', respectively.

Only $19.9 \%$ of overweight, $30.0 \%$ of obese and $21.8 \%$ of abdominally obese participants tried to lose weight, and nearly two-thirds of them $(79 \cdot 6,69 \cdot 5$ and $77.5 \%$, respectively) did not take any measures to manage their weight. Participants were more likely to choose the methods of 'Reduce the total diet' and 'Physical exercise' to lose weight (overweight participants: $66 \cdot 2 \%$ reduced their total diet and $52.6 \%$ did physical exercise; obese participants: $65.8 \%$ reduced their total diet and $56.0 \%$ did physical exercise; abdominally obese participants: $65 \cdot 8 \%$ reduced their total diet and $53.6 \%$ did physical exercise). More than half of participants had no body weight changes $( \pm 2.5 \mathrm{~kg})$ in the past 12 months (overweight: 61.0\%; obese: 57.1\%; abdominally obese: $60.5 \%$ ). The related lifestyles in these three groups were observed as follows: more than half of participants had a balanced diet (i.e. meat and vegetables in fine match); two-thirds had regular diet; more than $85 \%$ of participants were more likely to eat rice as staple food; and less than half of participants had a high salt intake. More than $80 \%$ of participants had breakfast every day. About $40 \%$ of the participants rarely or never did exercise; more than $70 \%$ of them chose to exercise around the house; and nearly $50 \%$ of participants chose to drive or go out by bus.

Table 3 shows the associations between sociodemographic factors and body weight misperception by multivariable logistic regression among individuals with overweight, obesity and abdominal obesity in Jilin Province. Rural residents were more likely to have misperception of their high body weight compared with urban residents ( $\mathrm{OR}=$ 1.340; 95\% CI 1.191, 1.509). Middle-aged participants $(\mathrm{OR}=1.826 ; 95 \%$ CI $1.605,2.078)$ and old participants $(\mathrm{OR}=3 \cdot 101 ; 95 \%$ CI 2.648, 3.632) were more likely to develop body weight misperception than youth. The prevalence of body weight misperception declined with the increase of education level (junior middle school: $\mathrm{OR}=0.628$; $95 \%$ CI $0.545,0.723$; senior middle school: OR $=0.498 ; 95 \%$ CI $0.426,0.583$; undergraduate and above: $\mathrm{OR}=0.395 ; 95 \%$ CI $0 \cdot 320,0 \cdot 487)$.

\section{Discussion}

We come to the conclusion that body weight misperception is common among adults from Jilin Province. Nearly half of overweight individuals did not realize that their weight had already reached the warning level. Only $29.1 \%$ of obese participants perceived themselves as being 'too fat'; instead, $10 \cdot 0 \%$ of those who were obese thought that they had 'normal weight' and $1.8 \%$ even thought that they were 'very thin'. Nearly $30.0 \%$ of centrally obese men and women perceived that their waist circumference was about right and they were of normal weight, and $5.7 \%$ of centrally obese men and women even perceived themselves as 'very thin'. A cross-sectional study that recruited 162 Chinese Americans in a community health centre in New York reported that $32 \%$ of individuals did not perceive their body weight correctly. Nearly $20 \%$ of them underestimated their body weight and there were significant differences by gender ${ }^{(21)}$. These Chinese Americans had a proper positioning towards their body weight compared with the present study participants. Many studies have shown that body weight misperception is common, but the correct cognition of weight is the premise of weight control ${ }^{(22,24,26,33)}$.

By analysing weight management among our participants, we found that nearly two-thirds of those with high weight did not take any measures to manage their weight. They were more likely to choose the methods of 'Reduce the total diet' and 'Physical exercise' to lose weight. More than half of high-weight participants had no body weight change $( \pm 2.5 \mathrm{~kg})$ in the past 12 months and most of them had a balanced diet (meat and vegetables in fine match). Two-thirds of them had regular diet, less than half of them had a high intake of salt and more than $80 \%$ of the participants had breakfast every day. Studies exploring the association between meal intake habit and abdominal obesity in four Spanish cities reported that having breakfast did not show any association with abdominal obesity, but the individuals who did not eat dinner were more susceptible to abdominal obesity ${ }^{(34)}$. This is consistent with our findings. Among our study participants, 
Table 2 Perceptions and behaviours towards overweight, obesity and abdominal obesity* among adults ( $n 20$ 552) aged 18-79 years from Jilin Province, Northeast China, 2012

\begin{tabular}{|c|c|c|c|c|c|c|c|c|c|c|c|c|c|c|c|c|c|c|}
\hline \multirow[b]{3}{*}{ Perceptions and behaviours } & \multicolumn{6}{|c|}{ Overweight participants } & \multicolumn{6}{|c|}{ Obese participants } & \multicolumn{6}{|c|}{ Abdominally obese participants } \\
\hline & \multicolumn{2}{|c|}{ Male } & \multicolumn{2}{|c|}{ Female } & \multicolumn{2}{|c|}{ Total } & \multicolumn{2}{|c|}{ Male } & \multicolumn{2}{|c|}{ Female } & \multicolumn{2}{|c|}{ Total } & \multicolumn{2}{|c|}{ Male } & \multicolumn{2}{|c|}{ Female } & & \\
\hline & $n$ & $\%$ & $n$ & $\%$ & $n$ & $\%$ & $n$ & $\%$ & $n$ & $\%$ & $n$ & $\%$ & $n$ & $\%$ & $n$ & $\%$ & $n$ & $\%$ \\
\hline Body weight perception & & & & & & & & & & & & & & & & & & \\
\hline Very thin & 173 & 4.6 & 218 & 5.0 & 391 & 4.8 & 27 & 1.4 & 44 & $2 \cdot 3$ & 71 & 1.8 & 253 & 4.8 & 416 & 6.8 & 669 & 5.7 \\
\hline Normal weight & 1643 & 44.5 & 1213 & 28.9 & 2856 & 37.4 & 181 & $11 \cdot 1$ & 160 & 8.5 & 341 & $10 \cdot 0$ & 1655 & 32.9 & 1569 & 26.5 & 3224 & 30.0 \\
\hline Overweight & 1551 & 48.1 & 2061 & $59 \cdot 2$ & 3612 & $53 \cdot 1$ & 939 & 62.8 & 879 & $54 \cdot 1$ & 1818 & $59 \cdot 1$ & 2263 & $51 \cdot 7$ & 2594 & $52 \cdot 0$ & 4857 & 51.8 \\
\hline Too fat & 78 & $2 \cdot 8$ & 203 & 7.0 & 281 & 4.7 & 336 & 24.7 & 471 & $35 \cdot 0$ & 807 & 29.1 & 414 & $10 \cdot 7$ & 637 & 14.7 & 1051 & 12.5 \\
\hline Weight management & & & & & & & & & & & & & & & & & & \\
\hline On diet & 555 & $17 \cdot 8$ & 711 & $22 \cdot 4$ & 1266 & 19.9 & 422 & 29.6 & 430 & $30 \cdot 7$ & 852 & $30 \cdot 0$ & 909 & 21.4 & 1013 & $22 \cdot 3$ & 1922 & 21.8 \\
\hline Gain weight & 22 & 0.8 & 8 & 0.2 & 30 & 0.5 & 6 & 0.3 & 5 & 0.5 & 11 & 0.4 & 39 & 1.0 & 19 & 0.5 & 58 & 0.7 \\
\hline No measures & 2891 & 81.4 & 3010 & 77.4 & 5901 & 79.6 & 1062 & $70 \cdot 1$ & 1132 & $68 \cdot 8$ & 2194 & 69.5 & 3667 & $77 \cdot 6$ & 4237 & $77 \cdot 2$ & 7904 & 77.5 \\
\hline Weight-loss measures & & & & & & & & & & & & & & & & & & \\
\hline Reduce the total diet & 313 & $56 \cdot 4$ & 529 & 75.5 & 842 & $66 \cdot 2$ & 254 & 60.0 & 316 & $73 \cdot 3$ & 570 & $65 \cdot 8$ & 522 & $57 \cdot 6$ & 749 & $75 \cdot 1$ & 1271 & 65.8 \\
\hline Low-fat diet & 89 & $15 \cdot 8$ & 116 & $15 \cdot 5$ & 205 & 15.7 & 77 & $17 \cdot 1$ & 81 & 17.9 & 158 & 17.4 & 162 & $17 \cdot 2$ & 183 & $16 \cdot 7$ & 345 & $16 \cdot 9$ \\
\hline Low-caloric diet & 59 & 10.4 & 79 & 11.4 & 138 & 10.9 & 56 & 13.0 & 55 & $15 \cdot 8$ & 111 & 14.2 & 109 & $11 . \overline{5}$ & 126 & 13.5 & 235 & 12.4 \\
\hline Physical exercise & 352 & 61.8 & 319 & 43.8 & 671 & $52 \cdot 6$ & 262 & $63 \cdot 3$ & 200 & $46 \cdot 4$ & 462 & $56 \cdot 0$ & 566 & $61 \cdot 3$ & 464 & 44.7 & 1030 & 53.6 \\
\hline Drug & 8 & 1.5 & 42 & 6.2 & 50 & 3.9 & 12 & 4.2 & 39 & $12 \cdot 1$ & 51 & 7.6 & 19 & 2.8 & 72 & 9.0 & 91 & 5.7 \\
\hline Others & 5 & 0.1 & 13 & 0.4 & 18 & 0.3 & 2 & 0.1 & 10 & 0.6 & 12 & 0.3 & 6 & 0.1 & 18 & 0.4 & 24 & 0.2 \\
\hline Body weight change & & & & & & & & & & & & & & & & & & \\
\hline Gain weight $(\geq 2.5 \mathrm{~kg})$ & 545 & $17 \cdot 6$ & 663 & $18 \cdot 7$ & 1208 & $18 \cdot 1$ & 274 & $20 \cdot 1$ & 332 & $23 \cdot 7$ & 606 & $21 \cdot 6$ & 769 & $18 \cdot 2$ & 912 & $19 \cdot 0$ & 1681 & 18.6 \\
\hline Flat $( \pm 2.5 \mathrm{~kg})$ & 2255 & $62 \cdot 7$ & 2268 & 58.9 & 4523 & 61.0 & 899 & $57 \cdot 6$ & 914 & 56.5 & 1813 & 57.1 & 2936 & $61 \cdot 6$ & 3211 & $59 \cdot 2$ & 6147 & 60.5 \\
\hline Lose weight ( $\geq 2.5 \mathrm{~kg}$ ) & 605 & $17 \cdot 7$ & 706 & 19.6 & 1311 & 18.6 & 289 & 19.8 & 281 & 17.6 & 570 & 18.9 & 816 & 17.9 & 1001 & 19.0 & 1817 & 18.4 \\
\hline Unconcern & 63 & 2.0 & 92 & 2.8 & 155 & $2 \cdot 3$ & 28 & 2.6 & 40 & $2 \cdot 2$ & 68 & 2.4 & 94 & 2.3 & 145 & $2 \cdot 8$ & 239 & 2.5 \\
\hline Diets & & & & & & & & & & & & & & & & & & \\
\hline Balance & 2235 & 65.4 & 2031 & $56 \cdot 3$ & 4266 & $61 \cdot 2$ & 987 & 67.5 & 853 & $57 \cdot 7$ & 1840 & 63.3 & 2959 & $65 \cdot 2$ & 2806 & 55.6 & 5765 & $60 \cdot 8$ \\
\hline Meat more & 484 & 15.4 & 157 & 4.7 & 641 & $10 \cdot 6$ & 241 & 17.0 & 78 & 5.4 & 319 & $12 \cdot 0$ & 691 & $16 \cdot 4$ & 216 & 4.7 & 907 & $11 \cdot 1$ \\
\hline Vegetable more & 749 & 19.3 & 1541 & $39 \cdot 0$ & 2290 & 28.2 & 262 & $15 \cdot 6$ & 636 & $36 \cdot 9$ & 898 & $24 \cdot 6$ & 965 & 18.4 & 2247 & 39.6 & 3212 & $28 \cdot 1$ \\
\hline Regular diet & & & & & & & & & & & & & & & & & & \\
\hline Yes & 2846 & $80 \cdot 0$ & 3151 & $82 \cdot 7$ & 5997 & $81 \cdot 2$ & 1323 & 83.0 & 2494 & $78 \cdot 7$ & 1171 & 75.6 & 3748 & $78 \cdot 7$ & 4496 & $83 \cdot 7$ & 8244 & 81.0 \\
\hline No & 622 & 20.0 & 578 & $17 \cdot 3$ & 1200 & 18.8 & 319 & 24.4 & 244 & 17.0 & 563 & 21.3 & 867 & $21 \cdot 3$ & 773 & $16 \cdot 3$ & 1640 & $19 \cdot 0$ \\
\hline Staple food & & & & & & & & & & & & & & & & & & \\
\hline Rice & 2965 & 87.3 & 3115 & 83.8 & 6080 & $85 \cdot 7$ & 1280 & 87.5 & 1305 & 84.6 & 2585 & $86 \cdot 3$ & 3909 & $86 \cdot 7$ & 4384 & 83.6 & 8293 & $85 \cdot 2$ \\
\hline Pasta & 359 & 9.2 & 400 & $10 \cdot 8$ & 759 & 9.9 & 157 & $9 \cdot 3$ & 171 & 10.0 & 328 & 9.6 & 507 & 9.7 & 555 & 10.4 & 1062 & 10.0 \\
\hline Cereal & 139 & 3.4 & 209 & $5 \cdot 3$ & 348 & 4.2 & 52 & 3.2 & 90 & 5.4 & 142 & 4.1 & 192 & 3.5 & 321 & 5.8 & 513 & 4.6 \\
\hline Others & 5 & 0.1 & 5 & 0.1 & 10 & 0.1 & 1 & 0.1 & 1 & 0.0 & 2 & 0.0 & 7 & 0.1 & 9 & 0.2 & 16 & 0.2 \\
\hline Sodium & & & & & & & & & & & & & & & & & & \\
\hline Appropriate & 1147 & 34.0 & 1237 & 33.7 & 2384 & 33.9 & 505 & 35.6 & 489 & 33.0 & 994 & 34.5 & 1547 & 34.5 & 1721 & 33.3 & 3268 & 33.9 \\
\hline Salty & 1592 & $46 \cdot 1$ & 1222 & 33.9 & 2814 & $40 \cdot 6$ & 732 & 48.4 & 590 & $36 \cdot 4$ & 1322 & 43.3 & 2159 & 47.0 & 1765 & 34.4 & 3924 & 41.3 \\
\hline Light & 729 & $19 \cdot 8$ & 1270 & 32.4 & 1999 & 25.5 & 253 & 15.9 & 488 & 30.6 & 741 & $22 \cdot 2$ & 909 & 18.5 & 1783 & $32 \cdot 3$ & 2692 & 24.8 \\
\hline Breakfast & & & & & & & & & & & & & & & & & & \\
\hline No & 552 & 18.9 & 493 & $15 \cdot 2$ & 1045 & $17 \cdot 2$ & 270 & 21.5 & 201 & 14.5 & 471 & 18.5 & 754 & $19 \cdot 7$ & 638 & $13 \cdot 9$ & 1392 & $17 \cdot 1$ \\
\hline Yes & 2916 & $81 \cdot 1$ & 3236 & 84.8 & 6152 & $82 \cdot 8$ & 1220 & 78.5 & 1366 & 85.5 & 2586 & 81.5 & 3861 & 80.3 & 4631 & $86 \cdot 1$ & 8492 & $82 \cdot 9$ \\
\hline Exercise & & & & & & & & & & & & & & & & & & \\
\hline Often & 1163 & $31 \cdot 1$ & 1245 & $30 \cdot 8$ & 2408 & 31.0 & 462 & $27 \cdot 1$ & 534 & 31.0 & 996 & 28.8 & 1577 & $30 \cdot 7$ & 1868 & 32.9 & 3445 & 31.7 \\
\hline Sometimes & 860 & 28.1 & 775 & $23 \cdot 6$ & 1635 & $26 \cdot 1$ & 439 & 34.2 & 300 & 21.8 & 739 & 29.0 & 1186 & $29 \cdot 3$ & 1026 & $22 \cdot 3$ & 2212 & $26 \cdot 1$ \\
\hline Rarely or never & 1445 & $40 \cdot 8$ & 1709 & $45 \cdot 6$ & 3154 & 43.0 & 589 & 38.6 & 733 & $47 \cdot 1$ & 1322 & $42 \cdot 2$ & 1852 & 40.0 & 2375 & 44.9 & 4227 & $42 \cdot 2$ \\
\hline Places of exercise & & & & & & & & & & & & & & & & & & \\
\hline In the home & 173 & 8.9 & 220 & $12 \cdot 0$ & 393 & $10 \cdot 2$ & 80 & 12.5 & 76 & 9.5 & 156 & 11.3 & 214 & 8.9 & 292 & $10 \cdot 8$ & 506 & 9.7 \\
\hline Around the house & 1561 & 75.5 & 1627 & $79 \cdot 1$ & 3188 & 77.0 & 660 & 68.5 & 697 & 83.0 & 1357 & $74 \cdot 1$ & 2126 & 75.0 & 2371 & 80.9 & 4497 & 77.5 \\
\hline Gymnasium & 289 & $15 \cdot 6$ & 173 & 8.9 & 462 & $12 \cdot 7$ & 161 & $19 \cdot 0$ & 61 & 7.5 & 222 & 14.5 & 423 & $16 \cdot 1$ & 231 & $8 \cdot 3$ & 654 & $12 \cdot 7$ \\
\hline Trip mode & & & & & & & & & & & & & & & & & & \\
\hline By bike & 526 & 13.2 & 507 & $13 \cdot 0$ & 1033 & $13 \cdot 1$ & 187 & 12.5 & 174 & $10 \cdot 3$ & 361 & 11.6 & 655 & 13.0 & 937 & $16 \cdot 3$ & 1292 & $12 \cdot 2$ \\
\hline Walking & 1131 & 32.1 & 1849 & 47.9 & 2980 & $39 \cdot 3$ & 449 & 28.9 & 771 & 47.4 & 1220 & $36 \cdot 8$ & 1552 & 31.9 & 1542 & 29.9 & 4264 & $40 \cdot 2$ \\
\hline By bus or driving & 1811 & 54.7 & 1373 & 39.0 & 3184 & 47.6 & 854 & 58.6 & 622 & 42.2 & 1476 & 51.6 & 2408 & 55.1 & 2557 & 53.7 & 4328 & 47.6 \\
\hline
\end{tabular}

${ }^{*}$ The numbers are unweighted, but the percentages are weighted. Complex weighted computation was used in the statistical analysis. 
Table 3 Multivariate analysis of risk factors associated with body weight misperception among high-weight adults $(n 11435)$ aged 18-79 years from Jilin Province, Northeast China, 2012

\begin{tabular}{lccc}
\hline Characteristic & $P$ & OR & $95 \% \mathrm{Cl}$ \\
\hline Residence & & & \\
$\quad$ Urban & $<0.001$ & 1.000 & Ref. \\
$\quad$ Rural & & & $1.191,1.509$ \\
Age & & 1.000 & Ref. \\
$\quad$ Youth & $<0.001$ & 1.826 & $1.605,2.078$ \\
$\quad$ Middle age & $<0.001$ & 3.101 & $2.648,3.632$ \\
$\quad$ Old people & & & \\
Education & & 1.000 & Ref. \\
$\quad$ Primary school and below & $<0.001$ & 0.628 & $0.545,0.723$ \\
$\quad$ Junior middle school & $<0.001$ & 0.498 & $0.426,0.583$ \\
$\quad$ Senior middle school & $<0.001$ & 0.395 & $0.320,0.487$ \\
$\quad$ Undergraduate and above & $<$ &
\end{tabular}

Ref., reference category.

about $40 \%$ of those with high weight rarely or never did exercise, more than $70 \%$ of them chose to exercise around the house and nearly $50 \%$ chose to drive or go out by bus. Evidence has suggested that regular self-weighing and weight management by nutrition professionals are more effective to lose weight ${ }^{(35,36)}$

Multivariate logistic regression analysis suggested that several factors were associated with body weight misperception. Compared with the youth, it was difficult for middle-aged and old participants to get a correct cognition of their high body weight. In our culture, symmetrical and slim features are regarded as symbols of health and beauty, but as people get older they do not pay particular attention to their appearance anymore, so they may not be consciously aware that they are gaining weight. Rural participants were more likely to get body weight misperception; possible explanations for this finding include that the education level of rural populations is lower than that of urban populations; also that rural inhabitants may lack information and knowledge about overweight and obesity.

The high prevalence of body weight misperception presents a severe challenge to public health in Jilin Province. Now we may safely arrive at the conclusion that redoubled efforts are needed to improve the situation of high weight and body weight misperception in Jilin Province. Change begins with attitude. Only when highweight individuals realize their problem can they take effective measures, like physical exercise and regular diet, to reduce the prevalence of high weight.

\section{Acknowledgements}

Acknowledgements: The authors thank all participants involved in this study. Financial support: This work was supported by the Scientific Research Foundation of the Health Bureau of Jilin Province, China (grant number 2011Z16). The funder had no role in the design, analysis or writing of this article. Conflict of interest: The authors declare no conflict of interest. Authorship: C.G., B.L., Y. Yu, Y. Yin and X.L. conceived and designed the experiments; C.G., X.L., Y. Yin, Y.S., P.Z., R.W., L.J. and Y.W. performed the experiments; C.G. analysed the data and drafted the manuscript; C.G. and X.L. participated in revising manuscript. Ethics of buman subject participation: The Ethics Committee of Jilin University School of Public Health approved this study (reference number 2012-R-011). Written informed consent was obtained from each participant.

\section{References}

1. Stevens GA, Singh GM, Lu Y et al. (2012) National, regional, and global trends in adult overweight and obesity prevalences. Popul Health Metr 10, 22.

2. Finucane MM, Stevens GA, Cowan MJ et al. (2011) National, regional, and global trends in body-mass index since 1980: systematic analysis of health examination surveys and epidemiological studies with 960 country-years and 9.1 million participants. Lancet 377, 557-567.

3. de Onis M, Blossner M \& Borghi E (2010) Global prevalence and trends of overweight and obesity among preschool children. Am J Clin Nutr 92, 1257-1264.

4. Popkin BM, Adair LS \& Ng SW (2012) Global nutrition transition and the pandemic of obesity in developing countries. Nutr Rev 70, 3-21.

5. Swinburn BA, Sacks G, Hall KD et al. (2011) The global obesity pandemic: shaped by global drivers and local environments. Lancet 378, 804-814.

6. Olshansky SJ, Passaro DJ, Hershow RC et al. (2005) A potential decline in life expectancy in the United States in the 21st century. $N$ Engl J Med 352, 1138-1145.

7. World Health Organization (2013) Follow-up to the Political Declaration of the High-level Meeting of the General Assembly on the Prevention and Control of Non-communicable Diseases. 66th World Health Assembly, Agenda Item 13.1, 27 May 2013. http://apps.who.int/gb/ebwha/pdf_files/ WHA66/A66_R10-en.pdf (accessed March 2017).

8. Ng M, Fleming T, Robinson M et al. (2014) Global, regional, and national prevalence of overweight and obesity in children and adults during 1980-2013: a systematic analysis for the Global Burden of Disease Study 2013. Lancet 384, 766-781.

9. Gortmaker SL, Swinburn BA, Levy D et al. (2011) Changing the future of obesity: science, policy, and action. Lancet 378, 838-847.

10. Ministries of Health and Science and Technology \& National Bureau of Statistics of the People's Republic of China (2015) The Nutrition and Health Status of the Chinese People. Beijing: People's Medical Publishing House.

11. Ma GS, Li YP, Wu YF et al. (2005) The prevalence of body overweight and obesity and its changes among Chinese people during 1992 to 2002. Chin J Prev Med 39, 311-315.

12. Zhai FY, Du SF, Wang ZH et al. (2014) Dynamics of the Chinese diet and the role of urbanicity, 1991-2011. Obes Rev 15, Suppl. 1, 16-26.

13. Ng SW, Howard AG, Wang HJ et al. (2014) The physical activity transition among adults in China: 1991-2011. Obes Rev 15, Suppl. 1, 27-36.

14. Yang Z, Xing X, Xiao J et al. (2013) Prevalence of cardiovascular disease and risk factors in the Chinese population with impaired glucose regulation: the 2007-2008 China national diabetes and metabolic disorders study. Exp Clin Endocrinol Diabetes 121, 372-374. 
15. Wu S, Wang R, Jiang A et al. (2014) Abdominal obesity and its association with health-related quality of life in adults: a population-based study in five Chinese cities. Health Qual Life Outcomes 12, 100.

16. Bacopoulou F, Efthymiou V, Landis G et al. (2015) Waist circumference, waist-to-hip ratio and waist-to-height ratio reference percentiles for abdominal obesity among Greek adolescents. BMC Pediatr 15, 50.

17. Kelishadi R, Mirmoghtadaee P, Najafi $\mathrm{H}$ et al. (2015) Systematic review on the association of abdominal obesity in children and adolescents with cardio-metabolic risk factors. J Res Med Sci 20, 294-307.

18. Wang F, Wild TC, Kipp W et al. (2009) The influence of childhood obesity on the development of self-esteem. Health Rep 20, 21-27.

19. Al-Hazzaa HM (2002) Physical activity, fitness and fatness among Saudi children and adolescents: implications for cardiovascular health. Saudi Med J 23, 144-150.

20. Gomes TN, Katzmarzyk PT, dos Santos FK et al. (2014) Overweight and obesity in Portuguese children: prevalence and correlates. Int J Environ Res Public Health 11, 11398-11417.

21. Liu S, Fu MR, Hu SH et al. (2016) Accuracy of body weight perception and obesity among Chinese Americans. Obes Res Clin Pract 10, Suppl. 1, S48-S56.

22. Zainuddin AA, Manickam MA, Baharudin A et al. (2014) Self-perception of body weight status and weight control practices among adolescents in Malaysia. Asia Pac J Public Health 26, 5 Suppl., 18S-26S.

23. Monteagudo C, Dijkstra SC \& Visser M (2015) Self-perception of body weight status in older Dutch adults. $J$ Nutr Health Aging 19, 612-618.

24. Agrawal P, Gupta K, Mishra V et al. (2014) A study on bodyweight perception, future intention and weightmanagement behaviour among normal-weight, overweight and obese women in India. Public Health Nutr 17, 884-895.

25. Deschamps V, Salanave B, Chan-Chee C et al. (2015) Bodyweight perception and related preoccupations in a large national sample of adolescents. Pediatr Obes 10, 15-22.

26. Klos LA \& Sobal J (2013) Marital status and body weight, weight perception, and weight management among US adults. Eat Behav 14, 500-507.
27. Jayawardena R, Byrne NM, Soares MJ et al. (2014) Body weight perception and weight loss practices among Sri Lankan adults. Obes Res Clin Pract 8, e192-e200.

28. Wang S, Kou C, Liu Y et al. (2015) Rural-urban differences in the prevalence of chronic disease in northeast China. Asia Pac J Public Health 27, 394-406.

29. Wang R, Zhang P, Gao C et al. (2016) Prevalence of overweight and obesity and some associated factors among adult residents of northeast China: a cross-sectional study. BMJ Open 6, e010828.

30. National Institutes of Health (1998) Clinical Guidelines on the Identification, Evaluation, and Treatment of Overweight and Obesity in Adults - The Evidence Report. Obes Res $\mathbf{6}$, Suppl. 2, 51S-209S.

31. Alberti KG, Eckel RH, Grundy SM et al. (2009) Harmonizing the metabolic syndrome: a joint interim statement of the International Diabetes Federation Task Force on Epidemiology and Prevention; National Heart, Lung, and Blood Institute; American Heart Association; World Heart Federation; International Atherosclerosis Society; and International Association for the Study of Obesity. Circulation 120, 1640-1645.

32. National Bureau of Statistics of China, Office for the Sixth Population Census of Jilin Province, Jilin Provincial Bureau of Statistics (2013) Tabulation on the 2010 Population Census of Jilin Province. Beijing: China Statistics Press.

33. Lee KM, Seo MS, Shim JY et al. (2015) Body weight status misperception and its association with weight control behaviours, depressive mood and psychological distress in nulliparous normal-weight young women. Ann Hum Biol 42, 528-532.

34. Keller K, Rodriguez Lopez S \& Carmenate Moreno MM (2015) Association between meal intake behaviour and abdominal obesity in Spanish adults. Appetite 92, 1-6.

35. Zheng Y, Klem ML, Sereika SM et al. (2015) Self-weighing in weight management: a systematic literature review. Obesity (Silver Spring) 23, 256-265.

36. Bleich SN, Bandara S, Bennett W et al. (2015) Enhancing the role of nutrition professionals in weight management: a cross-sectional survey. Obesity (Silver Spring) 23, 454-460. 